\title{
Notas del traductor de Reivindicaciones de la razón de Stanley Cavell
}

\author{
Diego Ribes \\ Departament de Filosofia \\ Universitat de València \\ Diego.Ribes@uv.es
}

Como habrá de resultar obvio a quien las lea, las notas que siguen están fuera de su lugar natural. Por tanto, algún tipo de justificación requiere su presencia aquí. Fueron redactadas para que aparecieran junto con la versión al castellano de Reivindicaciones de la razón de Stanley Cavell. Los editores, o alguien en su nombre, tras haber aceptado explícita y formalmente su inclusión en dicha traducción, tuvieron a bien suprimirlas sin haber recibido yo notificación alguna previa al evento de la publicación de la traducción. Hasta el presente, tras el evento, no se me ha dado tampoco ninguna explicación de esta decisión unilateral por parte de la editorial. Por lo demás, e infinitamente más importante, pienso que la Editorial Síntesis se ha tomado en serio la impresión del libro traducido, tarea ésta nada fácil dado lo intrincado del modo de escribir del autor; y pienso igualmente que el resultado ha sido una buena y cuidada edición. Si añadimos a esto el hecho de que, en el momento de la firma del contrato de traducción, Stanley Cavell era un filósofo prácticamente desconocido en nuestro país, y añadimos el hecho de la gran extensión del libro; y si consideramos, tomando los dos hechos juntos, el riesgo que esta traducción suponía para sus editores, creo que hay motivos de sobra para estar agradecidos a los responsables de la editorial y a los directores de la Colección Perspectivas por el esfuerzo realizado y el riesgo asumido.

Me ha parecido conveniente publicar aquí estas notas por varias razones. Primera, y más general, porque pienso que, dejando aparte ahora el valor intrínseco o conceptual que pudieran tener o no, podrían ser útiles como una ayuda para la lectura del libro. Segunda, más específica, porque algunas convenciones de las que me sirvo en la traducción -como, por 
ejemplo, la de poner entre paréntesis el término «claim» cuando no lo traduzco como «reivindicación»-podrían resultar ininteligibles para el lector, dado que extremos como éste quedan explicados en el texto de las notas. Y tercera, porque estas notas constituyen algo así como el precipitado de muchas discusiones habidas con el autor del libro, Stanley Cavell, quien fue quien sugirió en primer lugar un prólogo del traductor. Me parece, pues, que le debía la publicación de las mismas. Todas estas razones podrán verse apoyadas, eso espero, en el texto que sigue, y que transcribo aquí tal y como fue concebido y escrito para la traducción del libro, sin introducir cambio alguno.

Repito algunas de las cosas, pertinentes para lo que yo he de decir aquí, que se han dicho sobre la escritura (y, por tanto, sobre la filosofía) del autor: su modo de comunicación es a base de frases incompletas (T. Kuhn); el libro de Stanley Cavell es una obra clásica no sólo por todo lo que dice sino por todo lo que casi dice (J. Conant); se trata de un libro difícil de leer y oscuro (varios, el propio autor incluido); a veces da la impresión de que $S$. Cavell no quiere ser entendido, escribe de forma desmañada, soltando una afirmación tras otra sin ton ni son (varios, R. Rorty respecto a una parte, la central, de esta última opinión). La tarea que me propongo llevar a cabo a continuación, haciendo pie en estos testimonios, es describir algunos de los principales obstáculos que he encontrado en la traducción al castellano de la obra cumbre de Stanley Cavell The Claim of Reason, desde ahora, en castellano, Reivindicaciones de la razón. Si se tiene en cuenta que las dificultades a las que se alude en estas líneas lo son, o lo fueron cuando se publicó el libro, para compatriotas del autor, gente que habla su misma lengua nativa, espero que no parezca exagerado, ni se vea como una excusa de mis errores de traducción, si digo que las dificultades de su prosa filosófica, a la hora de verterla al castellano, alcanzan a veces el nivel de lo intraducible.

Empecemos por la palabra «claim» que aparece en el título. El problema de fondo, nada extraño por otra parte, es que no existe una sola palabra en castellano que capte el sentido pleno, todo el sentido o sentidos, que Cavell parece darle a este término. Lo extraño empieza a manifestarse cuando ninguna ( $\mathrm{y}$ he ensayado mu- 
chas) te parece medianamente satisfactoria, la palabra elegida deja indefectiblemente fuera un aspecto o sentido que crees igualmente importante; dicho de otro modo, encuentro fuertes reparos a todas las palabras castellanas que yo mismo he podido imaginar, y otros me han sugerido, como candidatas a la traducción de «claim», una palabra inglesa decididamente «humilde», a ras de suelo (como algo opuesto a las palabras típicamente «filosóficas» que tienden a ser «categoriales»). En resumen: necesitaría varias palabras para traducir este término. Durante un tiempo quise salvar a toda costa el aspecto o connotación «lingüística» de la palabra: llamada, clamor, (re)clamación, fueron algunos de los vocablos castellanos que se me ocurrieron. Al final tuve que abandonar mi empeño y las desestimé todas (no voy a exponer ahora las razones que me llevaron a esta decisión, me gustaría ser breve en estas notas, y ceñirme sólo a los aspectos que, creo, pueden ayudar a la mejor comprensión o seguimiento del texto traducido). Pero, tal vez, resulte ilustrativo decir por qué rechacé una de ellas, la que más llegó a tentarme: «reclamación» posee un sentido demasiado legalista que, aunque literalmente correcto, no tiene mucho que ver con los usos que de «claim» hace nuestro autor. Fui llevado de este modo a pensar en palabras cuyo importe principal es conceptual o abstracto: aspiración, pretensión, demanda, reivindicación fueron, quizás, las principales; y la elección final recayó en esta última, «reivindicación». Y así es como quedó el primer título que mantuvo cierta estabilidad durante bastante tiempo: La reivindicación de la razón. Me pareció que «reivindicación» subsumía en cierto modo el significado (parcial, pero relevante) de las otras palabras castellanas de este grupo. Alguien puede aspirar a algo pero no reivindicarlo, pero difícilmente al revés. $\mathrm{Y}$ alguien podría pretender algo y no llegar a reivindicarlo; podría, por ejemplo, robarlo. Por lo dicho hasta aquí podríamos hacer ya, creo, la siguiente recopilación que quiere transmitir el significado que yo le he dado a «reivindicación» tal y como aparece en el título del libro: una afirmación, sentencia, o expresión, que alguien propone con la pretensión de, o aspiración a, que sea conocimiento, o que se convierta en tal. Las otras modificaciones que he introducido en castellano respecto al título original pueden entenderse ahora, espero, sin demasiados reparos. En 
primer lugar, mi conversión de la palabra en plural quiere sugerir el hecho, ya señalado, de que «claim» tiene más de un significado o sentido, que tiene varios significados, o significados plurales. En segundo lugar, suprimir el artículo que precede a «reivindicación» en el original, introduciendo así cierta ambigüedad en el título, quiere enfatizar el hecho de que tal variedad de sentidos no es meramente cuantitativa, que las diferencias entre sus sentidos lo son de clase, o cualidad. No se trata, pues, simplemente de que la razón tenga muchas reivindicaciones que hacer, sino además que éstas pueden ser de muchas clases, algunas de ellas bastante extrañas para la propia «Razón», como, quizás, esas razones del corazón que, según Pascal, la razón no acierta a entender; o como, seguramente, el conocimiento que Otelo tiene de Desdémona, y que le es imposible admitir. Pascal y Otelo son traídos a colación aquí para recordar que la razón no sólo tiene reivindicaciones que hacer, sino también reivindicaciones que padecer (vuelvo enseguida sobre este punto). Estas últimas observaciones me llevan a otro conjunto de problemas que tengo con la palabra en cuestión, y que nos sacan de su mera aparición en el título del libro.

Me refiero ahora al hecho de que «claim» es, posiblemente, una de las palabras que más se repite a lo largo de todo el libro. Un libro que transita por distintas regiones de la filosofía, y que fue escrito en distintos períodos, bastante distantes entre sí, recibe su unidad, cohesión, y continuidad, por, entre otras cosas, el uso casi omnipresente del término «claim». Este efecto viene producido, o posibilitado, por un recurso del inglés del que carecemos en nuestra lengua. Cavell modula, o inflexiona, los distintos sentidos ya apuntados de esta palabra mediante proposiciones (además de la que aparece en el título «claim of», nos encontramos en el texto con «claim to», «claim for», «claim upon», «claim about»). Mi primer intento de traducción fue verter estas distintas modulaciones del sentido mediante una palabra castellana diferente cada vez (como hacemos normalmente con los «phrasal verbs»), sirviéndome de algunas de las palabras mencionadas en el párrafo anterior. El resultado fue un poco caótico, confuso: no se preservaba la mencionada cohesión y continuidad del texto de Cavell. Y de nuevo, como en el caso del título, tuve que rectificar este extremo y corregir 
la traducción. A este fin, he decidido conservar la palabra reivindicación del título a lo largo de todo el libro tanto como me ha sido posible, incluso algunas veces, en algunos contextos, donde la palabra resulta ligeramente forzada o artificial en castellano. Las otras veces, cuando resultaba muy forzada e incluso claramente extraña, he decidido traducirla por otra palabra castellana (aspiración, pretensión, e incluso afirmación). En estos casos, la mayor parte de las veces, he puesto entre corchetes, o paréntesis cuadrados, la palabra «claim» del texto original. Digo la mayor parte de las veces porque mi deseo era hacerlo así todas las veces, pero el texto original ya está por sí mismo plagado de signos de puntuación, y en particular de paréntesis y dobles paréntesis. Me pareció que excederme yo añadiendo todos los paréntesis cuadrados que me parecieran oportunos, podría hacer borroso un texto cuya escritura ya es de por sí muy complicada, si más no por su novedad. En fin, he decido introducir mis paréntesis cuadrados sólo en la medida que con ello me parecía quedar a salvo dicha cohesión y continuidad. Podría haber empleado otra convención al uso y para el mismo efecto sin emborronar el texto original: las notas a pie de página del traductor. Pero el autor ha decidido, en el presente libro al menos, prescindir él mismo de las notas a pie de página (a veces llamadas «críticas»), introduciendo la información normalmente destinada a ellas (referencias a otros libros, por ejemplo) en el cuerpo del texto. Para respetar, y para que quede patente, este rasgo de su escritura me he impuesto a mí mismo no hacer uso aquí de esta convención.

Aun así, a pesar de lo dicho, sigo manteniendo reservas con la traducción de «claim» por reivindicación. Me parece que nuestra palabra sigue siendo, en el aspecto que capta de «claim» que es correcto, demasiado..., cómo decirlo, bizarra. Me recuerda todavía demasiado esa arrogancia de la razón sin límites que pretendía poderlo (por ejemplo, demostrar) todo. Por ello, permítaseme terminar estas notas sobre el término «claim» con la siguiente observación. Creo que a este fin, y quizá sólo a este fin, podríamos reunir los sentidos de nuestro término en inglés, como es empleado por nuestro autor, en dos grupos principales, sin excluir con ello los distintos matices o aspectos que pudieran darse dentro 
de cada grupo. Un primer grupo de sentido se referiría a aquellas palabras que tienen que ver con el aspecto «activo» del conocimiento: la razón reivindica algo respecto de algo (en particular, y en nuestro caso, reivindica que una creencia o sentencia constituye conocimiento). Este es el sentido principal de reivindicación en castellano. Y en el libro de Cavell, es el sentido y significado predominantes que adquiere dicho término en la segunda parte del mismo, dedicado al estudio de la epistemología moderna y el escepticismo (lo que no quiere decir que este sentido no se encuentre en otras partes del libro). Si tenemos en cuenta ahora el lugar central que el escepticismo ocupa en la obra de Cavell, espero que se entienda algo más mi elección del término reivindicación como traducción de «claim». En todo caso, ésta fue una poderosa razón que me ayudó a tomar la decisión de escoger esta palabra castellana entre las otras candidatas a las que he aludido. El segundo grupo de sentido, e igualmente importante en el conjunto del libro $\mathrm{y}$, creo, más novedoso u original para nosotros, estaría compuesto por aquellas palabras, o mejor, como he sugerido, modulaciones de las palabras (en particular «claim») que tienen que ver o hacen alusión al aspecto «pasivo» del conocimiento. El término «pasión» es aquí (literalmente) paradigmático. Entonces se trata no ya de reivindicaciones que hace la razón sobre algo, exigiendo algo, sino de reivindicaciones que algunas cosas, lo otro de ella, le hacen o le imponen. Las reivindicaciones particulares que alguien puede hacerme a mí (por ejemplo en contextos éticos), o cosas que el cuerpo me pide, o las demandas que me hace (tal vez en forma de deseo), $\mathrm{o}$, más en general, las reivindicaciones que lo otro, la «otredad» (el mundo en general, los otros en general) hace sobre mí, a veces de forma muda, por el mero hecho de existir. Pero entonces, las reivindicaciones no lo son siempre de algo que no se tiene, sino que podrían serlo de algo que se posee quizá desde siempre, quizá por naturaleza, pero que hemos olvidado, perdido o reprimido. Dicho de otro modo, y con otra palabra candidata en otro tiempo a ser la traductora de «claim»: la razón no sólo «llama», sino que a veces «es llamada». Cavell señala, a nivel de su grafía, este sentido del término «claim», principalmente, adosándole la preposición «upon». (Y, desde luego, la distinción entre conocimiento activo y pasivo de la 
que me he servido en esta última observación es del propio Cave11). Y desde luego, el problema no queda completamente resuelto. El hecho perturbador para mí es que estos dos aspectos quizás no se den nunca por separado, en el caso del lenguaje entendido como humano, en el caso del conocimiento entendido como humano. Se enfatiza uno de los dos aspectos, pero el otro, no por no enfatizado no influyente, está igualmente presente. Pero ahora desemboco, creo, en un punto donde el traductor tendría que dejar paso al estudioso, comentarista o intérprete del libro de Cavell. Y ésta es una tarea que he decidido prohibirme severamente en estas notas.

Una cuestión que sí me parece propia de la competencia del traductor es decir algo sobre el empleo que hace Cavell de los términos «recognition» (o «recognizing») y «acknowledge» (o «acknowledgment»). El problema aquí es diferente, casi el opuesto, del que plantea el término «claim». Con este último nos encontrábamos con una palabra inglesa para cuya traducción satisfactoria necesitábamos varias palabras castellanas. En el presente caso, nos encontramos con dos palabras inglesas y una sola castellana para su traducción: reconocer o reconocimiento. El problema de fondo reside en que el autor se apoya en, o presiona sobre, el término «acknowledgment» para hacer su propio (original) trabajo filosófico, cosa que no ocurre con «recognition». La palabra «acknowledgment» y la noción que nos transmite, como elaborada por el autor, constituye algo así como la firma, o un trazo importante de la misma, del proyecto filosófico de Stanley Cavell. La partícula «ac» añadida a «knowledge» sugiere en inglés, como me contó el propio Cavell, una intensificación del conocer, y, me gustaría poder añadir yo, representa una ampliación de la noción del mismo tal y como se encuentra en la epistemología moderna (al menos como queda elaborada filosóficamente por nuestro autor). Pero además, como reivindica él mismo (por ejemplo, en las primeras páginas del capítulo primero de su libro En busca de lo ordinario), el autor no propone «acknowledge» como una alternativa a «knowledge», lo que viene sugerido por el hecho de que la primera palabra contiene a esta segunda, al igual que ocurre con nuestra palabra castellana reconocer que incluye conocer. Por estas razones y motivos me viene impuesto traducir «acknowledge» $\mathrm{y}$ «acknowledgment» por re- 
conocer y reconocimiento. Entonces mi problema es, o lo fue, con «recognition» o «recognizing». Estos términos son (su traducción como reconocer y reconocimiento) de uso frecuente en la literatura filosófica de nuestro país (por ejemplo, en las Investigaciones filosóficas de Wittgenstein, sección 270). Esto me movió a traducirlos también como «reconocer»y «reconocimiento» esperando que el contexto hiciese el trabajo de distinguir dos nociones o conceptos vertidos por la misma palabra. No fue así. El resultado era más bien introducir uniformidad y, de nuevo, la confusión de ambos conceptos, y se anulaba con ello el carácter específico, idiosincrásico, de la noción de «reconocimiento» (acknowledgment) en la obra de Cavell, una especie de marca de fábrica, como he sugerido, de su pensamiento. De modo que tuve que tomar, otra vez, una decisión al respecto, esta vez más costosa debido a la familiaridad filosófica mencionada del término y al temor a la extrañeza que en el lector español, debido a esta familiaridad, pudiera producir traducirlo por otra palabra distinta de reconocimiento. La decisión ha sido la de traducir «recognition», «recognizing», por «identificación» e «identificar» (la mayor parte de las veces), otras, según la regla del contexto, por «discernir»y «discernimiento», y aún otras por admitir o aceptar (que es una de las acepciones que «reconocer» tiene en castellano). La idea era buscar un término castellano que tuviera alguna connotación cognitiva (a falta de uno, otro que reconocimiento, que incorporara la palabra conocimiento), y creo que identificación la tiene. Y otra vez, y desde luego, la base o inspiración para mi elección se encuentra en el texto de Cavell, quien equipara en muchos contextos «recognize» . . . etc., con «identificar». . . etc., e incluso alguna vez coloca juntas las dos palabras unidas, o separadas, por la partícula «o».

Otra expresión del autor sobre la que creo conveniente decir algo es «a best case» («un caso mejor»). El problema viene planteado aquí por la presencia del artículo indefinido acompañando a un superlativo, problema que no se presenta con «el caso mejor» (expresión también empleada en el libro). El artículo indefinido parece convertir a «mejor» (en castellano al menos) en un comparativo, y por tanto parece estar pidiendo el segundo término de la comparación («mejor que qué»). El primer intento fue traducir la 
expresión por un sinónimo: «un caso ideal», «excelente», «paradigmático», «ejemplar», fueron algunos de ellos («óptimo» no es posible porque este término se emplea en el libro en otros contextos, para otros propósitos, y por tanto con otro significado). Muy pronto desestimé esta solución porque me parecía que debilitaba la fuerza que deposita el autor en su noción de «casos mejores»; y digo «debilita» habida cuenta de que con el artículo definido («el caso mejor», o «los casos mejores») la traducción al castellano de esta expresión no ofrece dificultad alguna, y aparece repetidas veces a lo largo del libro [lo que hacía todavía menos aconsejable suprimir la expresión «un caso mejor», pues entonces se perdía, o diluía, la relación de un caso mejor con (la noción) de el caso mejor]. Así que me decidí por la traducción literal de la expresión, aunque resulte dura o forzada en castellano. No creo que llegue a ser incorrecta. Cabe imaginar la siguiente situación. Has salido a comprarte una camisa. Tras entrar en varias tiendas y ver un montón de camisas sin haber encontrado nada que te gustase, de vuelta a casa, cansado y decepcionado, exclamas «necesito una camisa mejor». Al parecer, tenías una idea (no me atrevo a decir una forma platónica) de la camisa que querías, y ninguna de las que has visto satisface, o se acerca bastante a tu idea. Y ésta es otra razón por la que he traducido la expresión de Cavell como «caso mejor»: su término posee un grado de generalidad o abstracción (a pesar del artículo indefinido) que, pienso, le viene prestado por «el caso mejor» que sería, siguiendo con la comparación, la idea o forma platónica, y por tanto inexistente sobre la faz de la tierra. (Debo la articulación de esta razón a una sugerencia hecha por el autor como respuesta a una de mis preguntas. Por supuesto, del ejemplo de la camisa y de su elaboración presente Cavell no tiene ninguna culpa). Y por último, una tercera razón que me llevó a esta traducción literal estriba en que, después de todo, «un caso mejor» es, en parte al menos, uno de los pocos términos técnicos de esta obra, y como es bien sabido, los términos técnicos son más tolerantes en lo que a dureza (incluso artificialidad) lingüística se refiere.

Una última nota de carácter más general que se aplica a lo largo de todo el texto. Se trata del frecuente uso (¿íntimo?) del pronombre de segunda persona (tú), y de modo más general de todo 
tipo de pronombres (él, yo, esto, eso) cuando y donde su grafía explícita no es necesaria, y a veces no conveniente, ni en inglés ni en castellano. El problema se me aparece del siguiente modo. Como es bien sabido, el uso de pronombres es mucho más abundante en inglés que en castellano, incluido esa especie de comodín lingüístico «it». En particular el pronombre neutro «this», dada su repetición constante, supone una dificultad, si no más embarazosa, para las traducciones a nuestro idioma en el sentido de que traducirlo siempre por «esto» haría la traducción muy pesada, poco elegante y fluida. Hasta aquí una característica del inglés con la que cualquier estudiante de este idioma puede estar familiarizado al terminar su primer curso de estudio, y que cualquier traductor al castellano resuelve fácilmente, por ejemplo no traduciendo los pronombres la mayor parte de las veces. Ni qué decir tiene que la escritura de Cavell no difiere, no puede hacerlo, en este aspecto de cualquier otro escrito en esta lengua. Pero además de este hecho concerniente meramente a la idiosincrasia, por decirlo así, del inglés, Cavell hace un uso de los pronombres que pertenece a la idiosincrasia de su escritura, otro trazo de su firma: constituye una de las características definitorias de la misma, de su estilo si se prefiere decirlo así. Lo que significa, al nivel que ahora estamos hablando (que sólo tiene, hago recordar, el propósito de facilitar la lectura del libro), una abundancia de pronombres en su escritura que, como acabo de decir, incluso en inglés es innecesaria. Naturalmente, esta característica de su escritura no es arbitraria y responde, como era de esperar, a su concepción del lenguaje ordinario y de la filosofía en él inspirada. No voy a insistir ahora en este aspecto de su filosofía y escritura (he hablado de las características de la escritura de nuestro autor en la introducción a la traducción castellana de su libro En busca de lo Ordinario, editado por Cátedra), sino proseguir centrándome en la tarea de «notas del traductor» del presente libro. Como es natural, Cavell sabe de la extrañeza que podría causar esta característica de su escritura, y algunas veces interrumpe el hilo de su exposición para advertir al lector: «hace falta decir [aquí]. . . 'esto', o 'él', o...». En particular, he aquí el argumento que él mismo ha ofrecido, alguna vez, ante una posible reacción de extrañeza ante su uso idiosincrásico 
del pronombre de segunda persona (quizá el caso filosóficamente más relevante, o llamativo): «A veces me vuelvo al lector en una especie de irritación o impaciencia que no estaría dispuesto a manifestar a nadie con quien no mantuviera una relación íntima». Y Wittgenstein ciertamente se sirve de la forma personal «tú». [Pero entonces, el hecho que haya producido tanta extrañeza, sobre todo al principio, este rasgo de la escritura de Cavell, a mí me suena a que el Wittgenstein de la academia no tiene mucho que ver con el Wittgenstein «real» : ¡Tan obsesionados como estamos, o hemos estado, en «reconstruir» (Kripke incluido) el argumento (o argumentos) subyacente a las Investigaciones filosóficas!, ¡como si el propio Wittgenstein hubiese sido incapaz de hacerlo, y tuviésemos que salvarle nosotros la cara!. (Pero aquí, otra vez el comentarista debe detenerse para dejar paso al traductor)]. Y el traductor termina esta última nota del siguiente modo. Mi tarea, y dificultad, en este punto ha consistido en conseguir un equilibrio entre los dos hechos lingüísticos arriba mencionados. Por una parte decidir cuándo el uso abundante de pronombres personales se debía al inglés como un idioma, y entonces darles el tratamiento habitual: suprimirlos las más de las veces en la traducción castellana. Por la otra, estar muy al tanto para captar cuándo había que contar la presencia de un pronombre personal como perteneciente a la característica mencionada de la escritura de Cavell, es decir como presencia pretendida por él, y entonces el tratamiento no ha sido suprimir ninguno de ellos en la traducción sino mantenerlos lo más posible, en vistas a enfatizar esta característica de su escritura. No estoy seguro de haber conseguido dicho equilibro de modo satisfactorio.

No puedo terminar estas notas sin dejar constancia de la ayuda recibida por Stanley Cavell en la revisión de la presente traducción de su libro. He recibido apoyo, y me he sentido acompañado por otras muchas personas en la realización de una tarea tan ardua y elusiva como lo es la traducción de esta inmensa obra. Creo que no es éste el lugar oportuno para nombrar siquiera algunas de ellas, porque éste no es un libro mío y me parece que podría estar fuera de lugar el apartado de los agradecimientos, o reconocimientos. Pero estoy seguro que más de uno verá aquí y allá la huella 
de alguna observación que un día me hiciera. En cuanto a Cavell, la atención y apoyo prestados han sido literalmente impagables, literalmente un lujo (¿sólo intelectual?) para mí. Al principio incluso sorprendentes. Una persona que sabes muy ocupada, y que imaginas requerida constantemente por solicitudes parecidas a las tuyas, contestaba prontamente, algunas veces de inmediato, a tus preguntas, como si contestar a ellas fuera lo más importante para él. Estaba asombrado. Nunca me ha presionado en ninguna dirección (mucho menos ha mostrado exigencia alguna sobre algún punto o cuestión particular de traducción), se limitaba a contestar y comentar mis preguntas o dudas, muchas veces tomándose la molestia y el trabajo de explicar sus respuestas, aduciendo nuevos ejemplos, y esto me daba una seguridad enorme en el camino o caminos de traducción elegidos. Durante todo el proceso, el libro, y el pensamiento contenido en él, pasó de ser un mero libro a convertirse en un acompañante entrañable durante todos esos meses; me gustaría que siguiese siendo así. A él, y a todos los que no puedo nombrar, gracias. 


\section{RESUMEN}

En estas notas el autor expone las dificultades que ha experimentado a lo largo de la traducción de Reivindicaciones de la razón de Stanley Cavell. En particular, respecto a la traducción de ciertos conceptos clave en la obra de Cavell: claim (reivindicación), acknowledge (reconocer), best case (caso mejor), y su idiosincrásico uso de los pronombres.

Palabras clave: Cavell, Reivindicaciones de la razón, reivindicación, reconocimiento.

\section{ABSTRACT}

In this notes the author exposes the difficulties that he experimented while he was translating Stanley Cavell's The Claim of Reason. In concrete, with some key concepts inside Cavell's work: claim, acknowledge, best case, and his idiosyncratic use of pronouns.

Key words: Cavell, The Claim of Reason, claim, acknowledgement. 
